\title{
Hybrid Nanoimprint-Soft Lithography with Sub-15 nm Resolution
}

\author{
Zhiwei Li, ${ }^{\dagger}$ Yanni Gu, ${ }^{\dagger}$ Lei Wang, ${ }^{\dagger}$ Haixiong Ge, ${ }^{,, \dagger}$ Wei Wu, ${ }^{*, \neq}$ Qiangfei Xia, ${ }^{\ddagger}$ \\ Changsheng Yuan, ${ }^{\dagger}$ Yanfeng Chen, ${ }^{\dagger}$ Bo Cui, ${ }^{\dagger}$ and R. Stanley Williams ${ }^{\ddagger}$
}

\begin{abstract}
Department of Materials Science and Engineering, National Laboratory of Solid State
Microstructure, Nanjing University, Nanjing 210093, People's Republic of China,

Hewlett-Packard Laboratories, Hewlett-Packard Company, Palo Alto,

California 94304, and Department of Electrical and Computer Engineering, University

of Waterloo, 200 University Avenue, West Waterloo, Ontario N2L3G1, Canada
\end{abstract}

Received February 16, 2009; Revised Manuscript Received April 15, 2009

\begin{abstract}
We developed a hybrid nanoimprint-soft lithography technique with sub-15 $\mathrm{nm}$ resolution. It is capable of patterning both flat and curved substrates. The key component of the technology is the mold, which consists of rigid features on an elastic poly(dimethylsiloxane) (PDMS) support. The mold was fabricated by imprinting a reverse image onto the PDMS substrate using a UV-curable low-viscosity prepolymer film. Patterns with sub-15-nm resolution were faithfully duplicated on a flat substrate without applying external pressure. Gratings at $200 \mathrm{~nm}$ pitch were also successfully imprinted onto the cylindrical surface of a single mode optical fiber with a $125 \mu \mathrm{m}$ diameter.
\end{abstract}

Nanoimprint lithography (NIL) has been demonstrated as a high-throughput and low cost lithographic technique with sub-10 nm resolution. ${ }^{1,2}$ It has significant potential for various fields, such as electronics, ${ }^{3}$ photonics, ${ }^{4}$ bit-patterned media, ${ }^{5}$ and biological applications. ${ }^{6}$ The mold (also called a "stamp" or "template" in soft lithography and in step and flash imprint lithography ${ }^{8}$ ) is the crucial component for NIL and is typically fabricated on a stiff material such as silicon, nickel, silicon dioxide, or quartz. Techniques such as optical interference lithography and electron beam lithography, followed by pattern transfer using reactive ion etching (RIE), are used to pattern the master mold. ${ }^{9}$ The use of hard materials for imprint molds has enabled high spatial resolution for NIL. Soft lithography has primarily been used for patterning features and for fabricating structures on the size scale of $500 \mathrm{~nm}$ and larger. ${ }^{7}$ In contrast to the rigid molds used in NIL, the most commonly used stamp material in soft lithography is the flexible polymer poly(dimethylsiloxane) (PDMS), which enables an intimate physical contact with substrates without applying external pressure. The extension of conventional soft lithography to produce sub- $100 \mathrm{~nm}$ scale features has been limited by the low elastic modulus of the commercial PDMS (Sylgard 184 PDMS) used for fabricating stamps..$^{10}$ A higher modulus (ca. $8 \mathrm{MPa}$ ) "hard-PDMS" (h-

\footnotetext{
* Corresponding authors: Haixiong Ge e-mail haixiong@nju.edu.cn, phone 86-25-63686053, fax 86-25-83595535; WeiWu e-mail wei.wu@hp.com, phone (650) 857-7255, fax (650) 236-9885.

Nanjing University.

* Hewlett-Packard Co.

${ }^{\S}$ University of Waterloo.
}

PDMS) was developed by Schmid et al. ${ }^{11}$ to achieve soft lithography with resolution into the sub-100 $\mathrm{nm}$ regime. Although the h-PDMS stamp worked well for replication, there were still limitations for its applications: (i) releasing it from the master caused cracking across the face of the stamp; (ii) external pressure was required to achieve conformal contact with a substrate, which created long-range, nonuniform distortions over the large areas of contact. ${ }^{10}$ To overcome these problems, Odom et al. ${ }^{10}$ developed a composite stamp composed of two layers: a thin h-PDMS layer supported by a thick flexible PDMS layer, which combined the advantages of a rigid layer to achieve highresolution pattern transfer and an elastic support to enable conformal contact. However, as discussed in ref 10, some drawbacks still remained in using h-PDMS as a patterning layer for the double layer composite stamp, such as cracking during the stamp fabrication step and degraded conformal contact with the substrate compared with the PDMS material normally used in soft lithography. ${ }^{10}$

The ability to pattern curved surfaces is desired in many fields, such as MEMS, electronic devices, and optics, ${ }^{7}$ such as the recent demonstration of the fabrication of a highperformance, hemispherical electronic eye camera by Ko et al. ${ }^{12}$ A major feature of soft lithography is the ability to pattern features on highly curved and other nonplanar surfaces. ${ }^{13,14}$ This type of patterning task is impossible to accomplish with a conventional rigid NIL mold.

We have developed a photocurable cross-linked siliconcontaining polymer for NIL molds and have demonstrated 
(a) Preparing elastic support

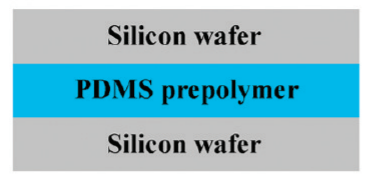

Elastic support treatmen

(b) Absorption of photo-curable resist

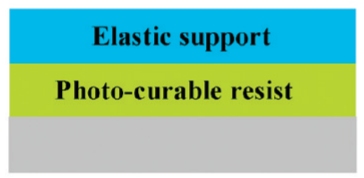

(c) Fabrication of hybrid mold
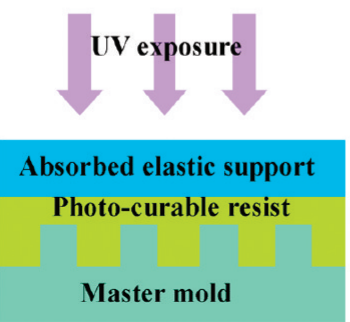

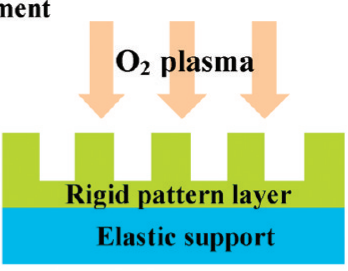

(e) UV imprint on curved substrate

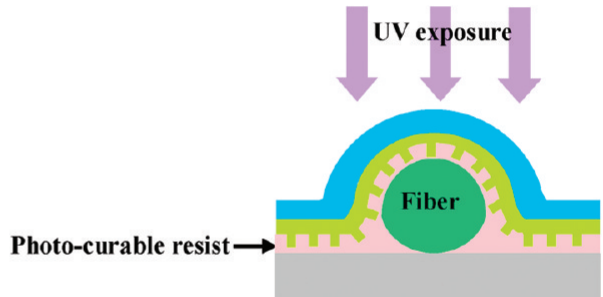

Releasing hybrid mold

(f) Imprinted patterns on curved substrate

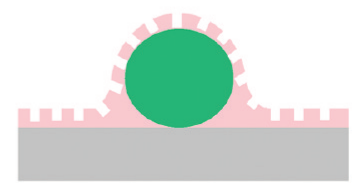

Figure 1. Schematic of the fabrication process of the hybrid mold and the hybrid nanoimprint-soft lithography: (a) preparing an elastic support; (b) absorption of the photocurable resist into the PDMS elastic support; (c) fabrication of hybrid mold by spin-coating nanoimprint resist on the master mold, making a conformal contact and curing the UV-curable resist; (d) mold surface treatment by $\mathrm{O}_{2}$ plasma oxidizing of the surface and coating with a mold release layer; (e) UV imprint on curved substrate by hybrid mold; and (f) imprinted patterns on curved substrate.

imprinted features smaller than $30 \mathrm{~nm} .{ }^{15}$ Such molds were fabricated on stiff substrates such as silicon dioxide, glass, and quartz. Here, we describe a mold composed of a UVcured rigid polymer used as the patterning template on an elastic PDMS support, which provided sub-15 nm spatial resolution free from cracks and fractures during conformal contact and mold release. The thin $(100-200 \mathrm{~nm})$ photocured feature layer and the thick $(\sim 2 \mathrm{~mm})$ elastic PDMS support were fused via an interpenetrating polymer network to create a mold that combined the advantages of a high-resolution nanoimprint mold and a conformal soft lithography stamp. Using this hybrid mold, we have fabricated nanoscale features on a highly curved surface by a simple nanoimprinting process.

The principles of the mold fabrication and the hybrid nanoimprint-soft lithography are summarized in Figure 1. First, an elastic support was prepared by casting and thermal curing of a PDMS prepolymer between two silicon wafers (Figure 1a), followed by a uniform absorption treatment that involved placing the elastic support onto a film of a siliconcontaining multifunctional photocurable resist spin-coated onto a silicon wafer (Figure 1b). The same silicon-containing photocurable nanoimprint resist was spin-coated onto a $\mathrm{Si}$ master mold that was not coated with a mold release agent. The absorbed elastic support was then placed against the resist-covered master mold and exposed to UV radiation in a nitrogen ambient (Figure 1c). After separation, the crosslinked photocurable resist on the master mold was transferred to the elastic support because of the difference in the surface adhesion properties. With a brief exposure to an $\mathrm{O}_{2}$ plasma, the surface of the duplicated mold was oxidized to form inorganic silica and was then coated with a mold release layer of trichloro $(1 \mathrm{H}, 1 \mathrm{H}, 2 \mathrm{H}, 2 \mathrm{H}$-perfluorooctyl)silane (Figure 1d). The resulting mold was ready to be used for hybrid nanoimprint-soft lithography (Figure 1e,f).

To prepare the elastic support, a liquid prepolymer of Sylgard 184 PDMS was degassed and cast between two silicon wafers that both had been coated with self-assembled monolayers (SAMs) of trichloro( $1 \mathrm{H}, 1 \mathrm{H}, 2 \mathrm{H}, 2 \mathrm{H}$-perfluorooctyl)silane. ${ }^{16}$ The cast was cured for $2 \mathrm{~h}$ at $80{ }^{\circ} \mathrm{C}$. The fluorinated SAM layer lowered the surface energy of the silicon wafers and enabled the elastic PDMS support to be easily released after the curing step. The area and thickness of the elastic support are determined by the size of silicon wafers and the amount of prepolymer utilized, respectively.

We used a photocurable reverse nanoimprint technique to fabricate the rigid cross-linked polymer features of the hybrid mold. A thin uniform liquid film of the photocurable resist was spin-coated onto the master mold rather than on the PDMS support to avoid swelling of the PDMS in the resist solution. The photocurable resist was composed of a lowviscosity UV-curable acrylated poly(dimethylsiloxane) material, a multifunctional acrylate cross-linker, and a free radical initiator. The mold surface was not coated with a releasing layer, because we required a uniform liquid film. The film 


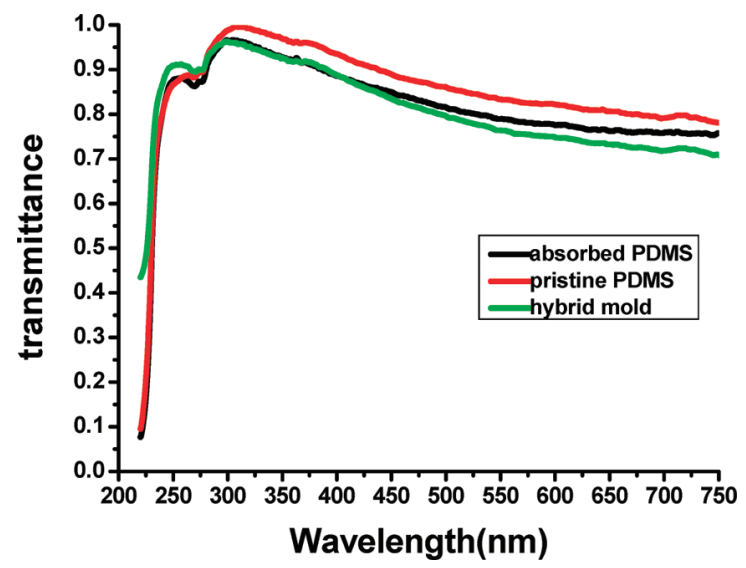

Figure 2. UV-vis transmission spectra of the pristine PDMS support (red), the PDMS support absorbed with the UV-curable resist (black), and the hybrid mold (green).

thickness was controlled from $10 \mathrm{~s}$ to $100 \mathrm{~s}$ of nm depending on the depth of the relief features on the master mold by the resist concentration and spin-coating speed. ${ }^{15}$ In our original mold fabrication process, an $\mathrm{O}_{2}$ plasma treatment of the PDMS was designed to convert the surface region of the organic polysiloxane to silica, and then an acrylate chlorosilane coupling agent, (3-acryloxypropyl)methyldichlorosilane, was employed to increase the adhesion between the PDMS substrate and the photocured features layer. ${ }^{15}$ However, an $\mathrm{O}_{2}$ plasma treatment can cause the spontaneous formation of disordered wavy patterns on the surface of the PDMS. ${ }^{17}$ To solve this problem, we took advantage of the intrinsic high permeability of PDMS for gases and organic molecules to absorb the elastic substrate with the multifunctional low viscous photocurable resist. The PDMS substrate was placed onto a $300 \mathrm{~nm}$ thick resist film that was spincoated onto a $\mathrm{Si}$ wafer. The photocurable molecules were capable of diffusing into the cross-linked network of thermalcured PDMS through the interaction of the resist film with the PDMS surface. After an equilibration time of absorption about $10 \mathrm{~min}$, the elastic support was separated from the $\mathrm{Si}$ wafer. The PDMS support absorbed with photocurable resist was then carefully placed onto the master mold spin-coated with a thin resist film. The soft and flexible properties of the PDMS enable it to make a conformal contact with the master mold over a large area without external pressure. The photocuring process was carried out in a nitrogen atmosphere because oxygen diffusing through PDMS can inhibit the freeradical polymerization of the photocurable resist. Ultravioletvisible (UV-vis) transmission spectra of a plain PDMS support and a PDMS support absorbed with the photocurable resist (Figure 2) show they are both transparent to broad band UV-vis light. The photocurable resist was exposed to UV radiation using a $\mathrm{Hg}$ arc lamp with an exposure of around $30 \mathrm{~mJ} / \mathrm{cm}^{2}$ through the transparent PDMS support. During the curing process, the resist spin coated onto the master mold not only self-polymerized but also reacted with the resist molecules absorbed into the PDMS to form an integrated interpenetrating polymer network near the surface region of PDMS support, which provided excellent adhesion of the two distinct layers. This strategy prevented the rigid features from peeling off the elastic support during the moldrelease process. Since the cured rigid layer based on acrylated PDMS had a low surface energy, the replicated mold was easily removed from the untreated master mold without any damage.

Although a water contact-angle measurement $\left(109^{\circ}\right)$ indicated that the surface of the hybrid mold was quite hydrophobic, the residual unreacted acrylate on the mold surface may further polymerize with an acrylate UV resist during imprintings and cause peeling of the mold. Thus a conventional antiadhesion layer of trichlorofluoroalkylsilane was also applied to coat on the mold for better mold release. In order to treat with the trichlorofluoroalkylsilane, the surface of the rigid polysiloxane layer was oxidized into inorganic silica by an $\mathrm{O}_{2}$ plasma $\left(48 \mathrm{~mW} / \mathrm{cm}^{2}, 25 \mathrm{mTorr}, 5\right.$ $\mathrm{sccm}, 5 \mathrm{~s}$ ). The low power and short time plasma treatment only oxidized the near-surface region of the mold but did not destroy the nanoscale features on the mold or form the irregular wavy patterns observed for bare PDMS substrate surfaces. The silanol groups were formed and exposed on the mold surface. The chlorosilane group of the trichlorofluoroalkylsilane release agent is capable of reacting with silanol groups of mold surface to form $-\mathrm{O}-\mathrm{Si}-\mathrm{O}-$ covalent bonds under catalysis of trace amounts of environmental water. ${ }^{18}$ The application of the mold release agent was carried out by putting the hybrid mold in the vapor phase of the chlorosilane under reduced pressure. ${ }^{16}$ The water contact angle on the rigid cross-linked polymer treated with $\mathrm{O}_{2}$ plasma and trichlorofluoroalkylsilane, which was $113^{\circ}$, was identical to that on a silicon dioxide substrate treated with trichlorofluoroalkylsilane..$^{15}$ As one of the key requirements, the mold must keep its release characteristics through repeated imprint operations. In initial experiments, we found that the hybrid polymer molds coated with an antiadhesion layer in this way could be used repeatedly more than 10 times without having to recoat the mold.

Although a relatively thick flexible PDMS support is used for the hybrid mold, it is still transparent enough to UV light to make it suitable for UV-NIL. To test the UV transmission properties of the hybrid mold, a $150 \mathrm{~nm}$ thick blank film of cross-linked UV resist was prepared on a 2-mm-thick PDMS support. The transmittance at $365 \mathrm{~nm}$ wavelength was over $93 \%$, and the transmittance for the $231-750 \mathrm{~nm}$ wavelength range was over $70 \%$ (Figure 2). Therefore, a photocurable imprint process is feasible using the hybrid polymer molds with either a broad band UV light source or a $\mathrm{Hg}$ i-line (365 $\mathrm{nm})$ source.

Several hybrid molds with nanoscaled features of various sizes and shapes were fabricated using master molds made by different lithography techniques (e.g., e-beam lithography, interference lithography, nanoimprint lithography, etc.). These hybrid molds were used for UV-NIL at room temperature without applying external pressure. The UV source used in this study was a $\mathrm{Hg}$ arc lamp (exposure around 30 $\mathrm{mJ} / \mathrm{cm}^{2}$ ). This photocuring process can be used in air as well as vacuum or a nitrogen atmosphere, since the rigid template used for patterning is a dense cross-linked polymer network that is resistant to permeation of oxygen, which inhibits the 

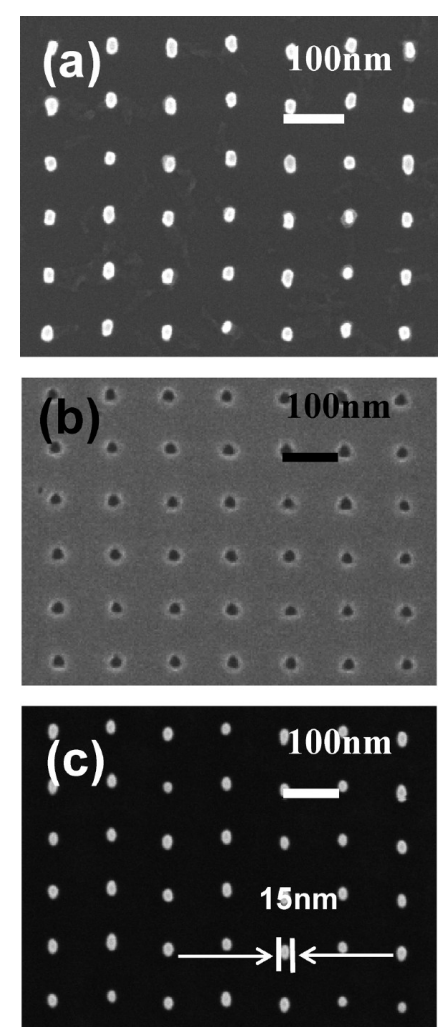

Figure 3. SEM images of the master mold, the replica and the imprint result of the replica (a) silicon dot array mold at $100 \mathrm{~nm}$ pitch and $15 \mathrm{~nm}$ diameter features, (b) the hybrid hole array mold, and (c) imprint result of the hybrid hole array mold.

free-radical polymerization of UV-curable resist. After photocuring, the hybrid mold can then be easily released from the substrate.

The mold fabrication process faithfully duplicated nanoscale features from the master molds. The molds were demonstrated in high-fidelity pattern transfer as well. For example, sub-15 nm resolution is shown in Figure 3. Parts $a$ and $b$ of Figure 3 are scanning electron microscopy (SEM) images of a master Si mold with a dot array at $100 \mathrm{~nm}$ pitch and $15 \mathrm{~nm}$ in diameter, and its hybrid mold replica on a PDMS support, respectively. Figure $3 \mathrm{c}$ shows that the 15 $\mathrm{nm}$ dot patterns were accurately replicated by the hybrid mold. The excellent fidelity of the results indicates that it should be possible to imprint features much smaller than 15 $\mathrm{nm}$. The resolution in our experiments was limited entirely by the feature size resolution of the master mold. For this example, the mold had a 4 in. diameter and the imprint was uniform over the entire area.

More importantly, the flexible nature of the hybrid molds makes them suitable for curved substrates. As a demonstration, a segment of a cylindrical single-mode optical fiber $(125 \mu \mathrm{m}$ in diameter) was placed on the surface of a $\mathrm{Si}$ wafer. The photocurable imprint resist was spin-coated onto the curved surface. A standard UV-NIL process using a hybrid mold with $200 \mathrm{~nm}$ pitch gratings was carried out on the $\mathrm{Si}$ wafer with the optical fiber. The $200 \mathrm{~nm}$ pitch gratings were duplicated onto the fiber surface with the grating line directions both perpendicular and parallel to the fiber axis. Looking at the inserted SEM image of the cross section of a patterned fiber (Figure
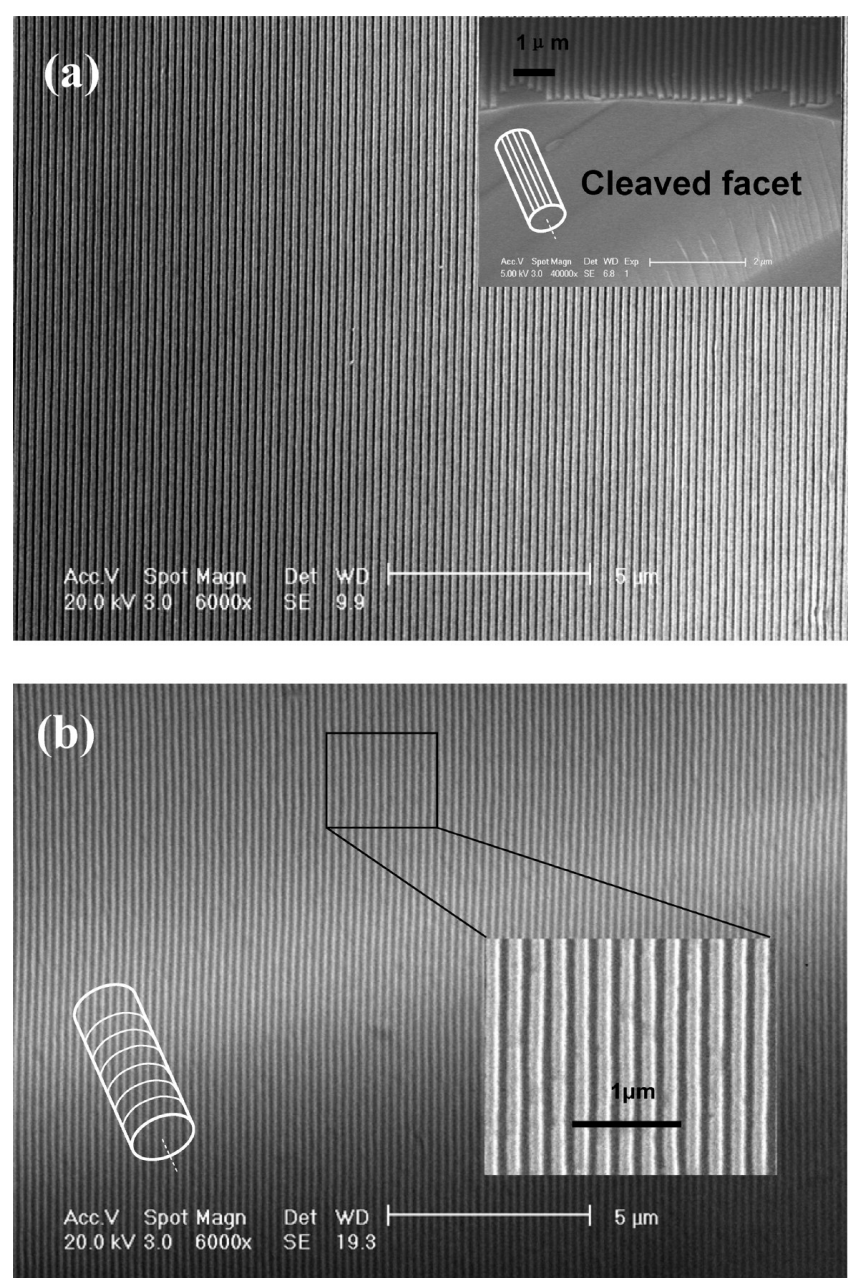

Figure 4. SEM image of $200 \mathrm{~nm}$ pitch grating on the surface of $125 \mu \mathrm{m}$ diameter optical fiber with the direction (a) parallel with and (b) perpendicular to the fiber axis. In (a), the cross-sectional view of the patterned fiber shows that the grating structures are distributed on a cylindrical surface parallel to the fiber.

4a), we see that the grating structures were uniformly distributed on the cylindrical surface parallel to the fiber. Several defects around edge of the cleaved facet were induced by fiber cutting for SEM imaging. Top views of the grating features parallel with and perpendicular to the fiber are shown in Figure 4a,b. The quality and uniformity are excellent, as the lines extend for several tens of micrometers without cracks or variation in period. The contrast of the dark and bright area of the top view images indicated that the grating patterns were not located on the same plane. In this case, the patterned area extended over $60^{\circ}$ around the fiber. The cylindrical surface with $125 \mu \mathrm{m}$ in diameter is highly curved, but there is no sign showing that it is the ultimate limit of substrate curvature to the hybrid mold.

The complementary mechanical properties of the rigid crosslinked patterning layer and elastic support of the hybrid mold are crucial factors for ideal imprinting results. The high mechanical strength of the rigid patterning layer is the key point to achieve high lithographic resolution. On the other hand, a soft and elastic support with high flexibility allows a conformal contact between mold and substrate without applying large imprint pressure. The mechanical properties of the two types 

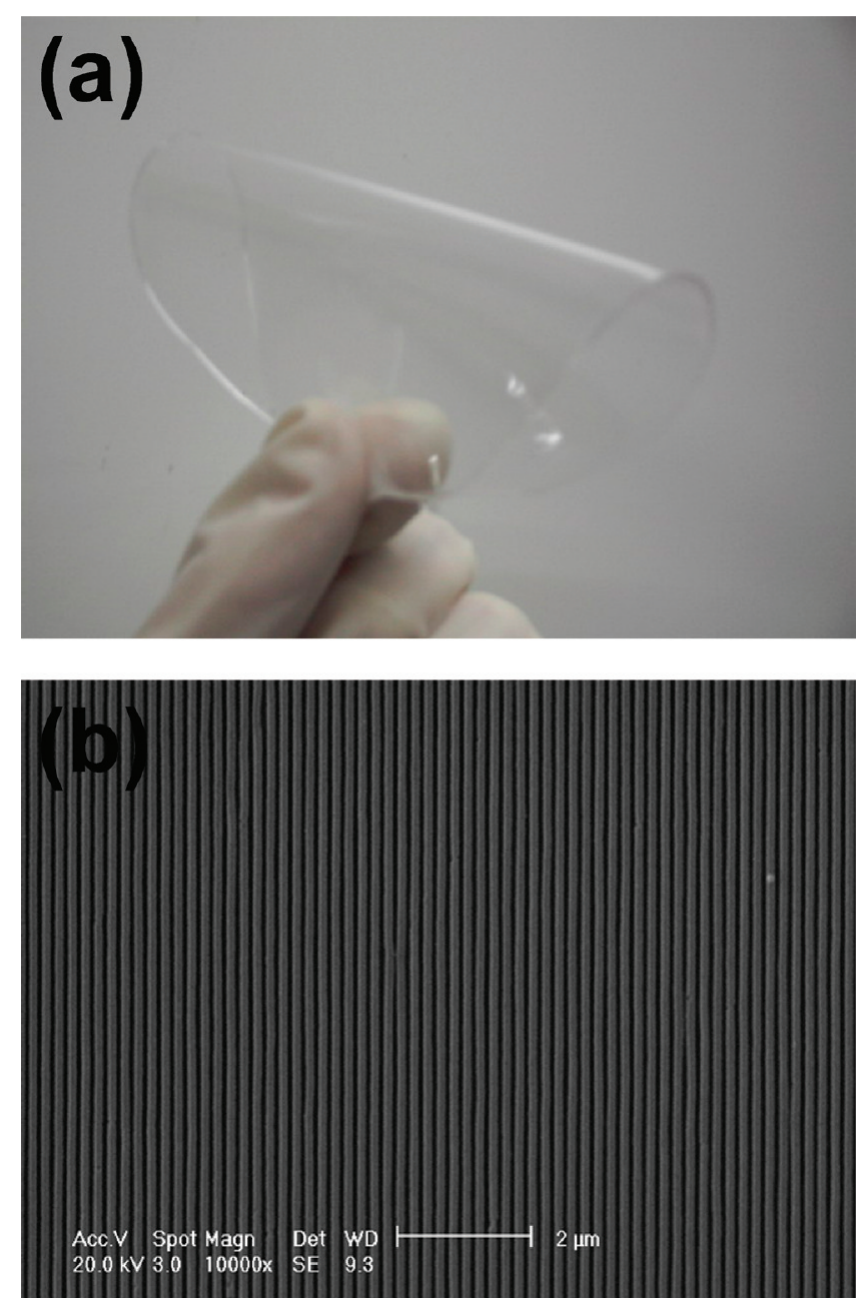

Figure 5. (a) Photograph of the hybrid mold with the $200 \mathrm{~nm}$ pitch grating bent over on itself. (b) SEM image of the $200 \mathrm{~nm}$ pitch grating replicated from the bent area of the hybrid mold.

of mold layers, rigid and flexible, were estimated by dynamical mechanical analysis (DMA) for the tensile modulus and elongation at breaking with the samples prepared by molding and curing pure liquid precursors. The tensile modulus can be calculated from the slope of the stress - strain curve in the linear region, and the elongation at breaking can be measured from the value of the strain at which fracture occurs, which corresponds to the abrupt decrease in the stress. ${ }^{19}$ The tensile modulus and elongation at breaking for the rigid patterning layer is $216 \mathrm{~N} / \mathrm{mm}^{2}$ and $1.3 \%$, respectively. Compared with the hard PDMS modulus of $\sim 9 \mathrm{~N} / \mathrm{mm}^{2}$, the much higher modulus of this patterning layer provided sufficient mechanical strength to sustain the high capillary forces and the nanoimprint pressure acting at the nanometer level. The modulus and the elongation at breaking of the flexible support $\left(2.4 \mathrm{~N} / \mathrm{mm}^{2}\right.$ and $\left.236 \%\right)$ are similar to these reported for sylgard 184 PDMS in other references, ${ }^{11}$ which enabled conformal contact with a substrate without external pressure. The mold with $200 \mathrm{~nm}$ pitch grating was bent along and across the grating lines at angles as large as those shown in Figure 5a. There were no apparent bending effects observed from the SEM images of the grating patterns (Figure $5 b$ ), which were replicated from the bent area of the hybrid mold.
In summary, we developed a hybrid nanoimprint and soft lithography mold composed of a rigid cross-linked patterning layer on an elastic PDMS support. This mold combines the advantages of both a rigid nanoimprint mold to achieve a highresolution pattern transfer and a flexible soft lithography stamp to enable conformal contact without applying an external pressure. An interpenetrating polymer network was formed between the interface of the UV-cured rigid patterning layer and the flexible PDMS substrate to provide excellent adhesion of the two distinct materials. We have demonstrated high-fidelity pattern transfer and sub-15 nm resolution using the hybrid molds. We also fabricated sub-100 nm features on the surface of an optical fiber. With the ability of NIL on highly curved and nonplanar surfaces, the hybrid molds are promising in applications in novel nanoelectronic and nanophotonic devices with nonplanar geometries.

Acknowledgment. This work was jointly supported by the National Basic Research Program of China (973 Program) (Grant No. 2007CB613202), the National Nature Science Foundation of China (Grant No. 50673040), and the National High Technology Research and Development Program of China (863 Program) (Grant No. 2007AA03Z334).

Supporting Information Available: Figures showing schematic of the polymer network, SEM images of the mold and imprint results, and stress-strain curves for the mold, This material is available free of charge via the Internet at http://pubs.acs.org.

\section{References}

(1) Chou, S. Y.; Krauss, P. R.; Zhang, W.; Guo, L. J.; Zhuang, L. J. Vac. Sci. Technol., B 1997, 15, 2897.

(2) Austin, M. D.; Ge, H. X.; Wu, W.; Li, M. T.; Yu, Z. N.; Wasserman, D.; Lyon, S. A.; Chou, S. Y. Appl. Phys. Lett. 2004, 84, 5299.

(3) Menard, E; Meitl, M. A.; Sun, Y. G.; Park, J. U.; Shir, D. J.; Nam, Y. S.; Jeon, S.; Rogers, J. A. Chem. Rev. 2007, 107, 1117-1160.

(4) Guo, L. J. J. Phys. D: Appl. Phys. 2004, 37, R123-R141.

(5) Oshima, H.; Kikuchi, H.; Nakao, H.; Itoh, K. I.; Kamimura, T.; Morikawa, T.; Matsumoto, K.; Umada, T.; Tamura, H.; Nishio, K.; Masuda, H. Appl. Phys. Lett. 2007, 91, 022508.

(6) Truskett1, V. N.; Watts, M. P. C. Trends Biotechnol. 2006, 24 (7), 312-317.

(7) Xia, Y. N.; Whitesides, G. M. Angew. Chem., Int. Ed. 1998, 37, 550575.

(8) Colburn, M. Proc. SPIE 1999, 3676, 379-389.

(9) Murphy, P. F.; Morton, K. J.; Fu, Z. L.; Chou, S. Y. Appl. Phys. Lett. 2007, 90, 203115.

(10) Odom, T. W.; Love, J. C.; Wolfe, D. B.; Paul, K. E.; Whitesides, G. M. Langmuir 2002, 18, 5314-5320.

(11) Schmid, H.; Michel, B. Macromolecules 2000, 33, 3042-3049.

(12) Ko, H. C.; Stoykovich, M. P.; Song, J. Z.; Malyarchuk, V.; Choi, W. M.; Yu, C. J.; Geddes, J. B.; Xiao, J. L.; Wang, S. D.; Huang, Y. G.; Rogers, J. A. Nature 2008, 454, 748-753.

(13) Jackman, R. J.; Wilbur, J. L.; Whitesides, G. M. Science 1995, 269, 664.

(14) Rogers, J. A.; Jackman, R. J.; Whitesides, G. M.; Wagener, J. L.; Vengsarkar, A. M. Appl. Phys. Lett. 1997, 70 (1), 7-9.

(15) Ge, H. X.; Wu, W.; Li, Z. Y.; Jung, G. Y.; Olynick, D. L.; Chen, Y. F.; Liddle, J. A.; Wang, S. Y.; Williams, R. S. Nano Lett. 2005, 5 (1), 179-182.

(16) Jung, G. Y.; Li, Z. Y.; Wu, W.; Chen, Y.; Olynick, D. L.; Wang, S. Y.; Tong, W. M.; Williams, R. S. Langmuir 2005, 21, 1158-1161.

(17) Bodas, D.; Khan-Malek, C. Sens. Actuators, B 2007, 123, 368-373.

(18) Chaudhury, M. K.; Whitesides, G. M. Langmuir 1991, 7, 1013.

(19) Yoo, P. J.; Choi, S. J.; Kim, J. H.; Suh, D.; Baek, S. J.; Kim, T. W.; Lee, H. H. Chem. Mater. 2004, 16, 5000-5005.

NL9004892 